\title{
Isolated Attosecond Pulses Generated Directly from a Femtosecond Chirped Pulse Amplifier
}

\author{
Yi Wu, Steve Gilbertson, Sabih Khan, Michael Chini, Kun Zhao, Ximao Feng and Zenghu Chang ${ }^{*}$ \\ J. R. Macdonald Laboratory, Department of Physics, Kansas State University, Manhattan KS 66506 \\ Email: "Chang@phys.ksu.edu
}

\begin{abstract}
Using a generalized version of double optical gating, we produced single isolated attosecond pulses with $2 \mathrm{~mJ}, 25 \mathrm{fs}$ driving lasers. Through attosecond streaking, we characterized isolated 160 attosecond pulses with $170 \mathrm{pJ}$ pulse energy.
\end{abstract}

(C) 2009 Optical Society of America

OCIS codes: (020.2649) Strong field laser physics; (320.7100) Ultrafast measurements

\section{Introduction}

Isolated attosecond pulses are important for current trends in cutting edge physics concerning electron dynamics in atoms, molecules, and solids. High order harmonic generation has recently been used to generate 80 as pulses [1]. However, the previously demonstrated gating methods required starting with short, few-cycle femtosecond laser pulses with precisely controlled carrier-envelope phase, few labs in the world have this technique. Recently, we developed a method called double optical gating (DOG) consisting of a combination of two-color gating and polarization gating. This technique allows laser pulses as long as 9 fs to generate isolated attosecond pulses [2]. Using a superposition of right and left elliptically polarized pulses in the double optical gating scheme reduces the leading edge ionization further than circularly polarized pulses. This so called generalized method of DOG (GDOG) allows single attosecond pulses to be generated from up to $28 \mathrm{fs}$ pulses [3]. This technique opens the door for more labs to join attosecond science research since sub 25 fs chirped pulse amplifiers (CPA) are commercially available. Here we characterized the temporal shape and phase of the single isolated attosecond pulses generated by GDOG technique directly from a 25 fs CPA.

\section{Experiments and discussions}

$25 \mathrm{fs}, 2 \mathrm{~mJ}$ pulses at $1 \mathrm{kHz}$ repetition rate were generated from a home built 14 pass CPA. A 300 micrometer quartz plate was used as a birefringent filter to compensate the gain narrowing effect which occurred in the amplification process and started with the output pulses from a 12 fs carrier envelope phase locked (CEP) oscillator. The slow CEP drift of the amplifier is compensated by feedback controlling the separation of the grating in the stretcher.



Fig.1. The setup for the measurement of attosecond pulse generation and measurement. One arm generates attosecond pulses while the other has the streaking IR field. The two beams are focused to a second gas target where the streaked photoelectrons are recorded with a time-of-flight detector. The green beam is used for locking the interferometer. BW, fused silica Brewster window.

The optics for GDOG consists of a pair of birefringent quartz plates, a fused silica window, and a type-1 BBO crystal. A diagram of the optics is shown in figure 1 . The input linearly polarized laser pulse is split into two orthogonal components by the first quartz plate at $\pm 45^{\circ}$ with respect to the input polarization. This plate also introduces a delay between the two pulses of a full number of optical cycles of the fundamental laser period. These two orthogonal components next pass through a $0.5 \mathrm{~mm}$ fused silica window oriented at the Brewster angle and used to reject a portion of the components of the two pulses lying along the axis parallel to the input polarization while leaving the ones along the other axis unchanged.

The modified pulses then pass through the second quartz plate and the BBO crystal together acting as a quarter waveplate. Since the pulses do not have equal magnitude electric field components along the slow and fast axes of the two birefringent plates, right and left elliptically polarized pulses are generated. The superposition of these gives the ellipticity dependent pulse containing a small linear portion, called the gate width, between them. Since the final plate is a BBO crystal, a weak linear second harmonic field is generated with its polarization parallel to the linear portion of the ellipticity dependent pulse. The purpose of the second harmonic is to break the symmetry of the gate width of the pulse thereby allowing harmonic emission only once per optical cycle. This means the gate width can be a full optical cycle and still only allow one attosecond pulse. 


\section{JThA1.pdf}

To temporally characterize the attosecond pulses, an interferometric streak camera was used as shown in figure 1. A linear 25 fs laser pulse is split by a broadband $80 / 20$ beam splitter with the majority of the beam being used for the GDOG attosecond pulse generation. The beam was then focused onto an argon gas target to generate the attosecond pulse. The attosecond pulse then passed through an aluminum filter to compensate the intrinsic chirp from the high harmonic generation process and also to filter out the residual fundamental laser. Meanwhile the reflected portion of the original laser pulse passed through a delay stage and recombined with the attosecond pulse at a hole drilled mirror and focused by a normal incidence Mo/Si mirror onto a second krypton gas jet. A continuous wave laser with $532 \mathrm{~nm}$ center wavelength co-propagating with the fundamental laser was used to temporally stabilize the interferometer. The temporal jitter was measured to be $\sim 8$ as.

Photoelectrons generated from krypton by attosecond pulse were then given a momentum shift from the fundamental laser. The photoelectron momentum spectrum is recorded with a position sensitive time-of-flight detector as a function of the relative delay which we introduce between the fundamental and attosecond pulses. Using the iterative principle components generalized projections algorithm (PCGPA), the attosecond pulse and phase could be reconstructed. Figure 2(a) and (b) show the experimental and reconstructed streaked spectrograms, respectively. The figures contain 10 cycles as this increases the accuracy of the reconstruction due to the redundant data. Figure 2(c) shows the temporal profile of the pulse (solid line) and the temporal phase (dashed line). The flat phase indicates a nearly transform limited pulse and the full width at half maximum (FWHM) of the pulse indicates 163 as duration. The transform limited pulse duration of this result is calculated as $\sim 162$ as. The inset figure shows the temporal profile over a several cycle range. This indicates that the contributions from pre and post pulses are less than $0.1 \%$ of the main peak demonstrating that the pulse is indeed an isolated attosecond pulse.
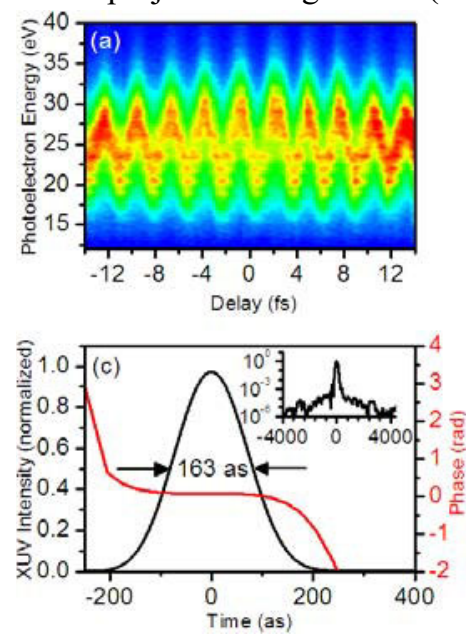

Fig. 2. Attosecond pulses from 25 fs lasers with GDOG. The experimental (a) and retrieved (b) streaked. The temporal profile (solid line) and temporal phase (dashed line) are shown in (c). The inset figure shows the same temporal profile but over an extended temporal range. Figure (d) shows the experimental (dotted line) and retrieved (solid line) XUV onlv spectrum.

Finally, the attosecond pulse energy was estimated to be $170 \mathrm{pJ}$ by scaling the result to previous results measured with an XUV photodiode. This attosecond pulse energy is limited by the input laser power, which in our case was $2 \mathrm{~mJ}$. However, if the GDOG method were applied to a joule pulse energy laser, the attosecond pulse energy could achieve previously unattainable level.

\section{Conclusions}

Using the GDOG technique, isolated attosecond pulses with energy of $\sim 170 \mathrm{pJ}$ were generated directly from a CPA producing $25 \mathrm{fs}, 2 \mathrm{~mJ}$ laser pulses for the first time. Temporal characterization of the attosecond pulses showed $\sim 163$ as pulses were generated. The GDOG technique will allow many labs to generate single attosecond pulses of XUV photons directly from an amplifier which should help further expand the field of attosecond science. This material is supported by the U. S. Department of Energy, the NSF under Grant No. 0457269, and by the U. S. Army Research Office under Grant No. W911NF-07-1-0475.

\section{References}

[1] E. Goulielmakis, M. Schultz, M. Hofsetter, V.S. Yakovlev, J. Gagnon, M. Uiberaker, A.L. Aquila, E.M. Gulikson, D. T. Attwood, R. Kienberger, F. Krausz, and U klienberg, Science 320, 1614 (2008).

[2] H. Mashiko, S. Gilbertson, C. Li, S. D. Khan, M. M. Shakya, E. Moon, and Zenghu Chang, Phys. Rev. Lett. 100,103906 (2008).

[3] X. Feng, S. Gilbertson, H. Mashiko, H. Wang, S. Khan, M. Chini, Y. Wu, K. Zhao, and Z. Chang, Phys. Rev. Lett. 103, 183901 (2009). 